\title{
THE IMPOSSIBILITY OF FANO'S CONFIGURATION IN A PROJECTIVE PLANE WITH EIGHT POINTS PER LINE ${ }^{1}$
}

\author{
WILLIAM A. PIERCE
}

1. Introduction. R. C. Bose and W. L. Stevens have shown independently $[1 ; 2]$ that the existence of a projective plane with $n+1$ points per line is equivalent to the existence of a completely orthogonal $n$-by- $n$ Latin square. In 1939, H. W. Norton [3] gave a statistical enumeration of all the 7-by-7 Latin Squares. On the basis of this enumeration, Bose and Nair [4] observed that the only completely orthogonal 7-by-7 squares are those associated with the Desarguesian plane having eight points per line. In 1951, A. Sade [7] noted an omission in Norton's list, although the omitted squares do not contribute any new completely orthogonal systems. Strictly speaking, no complete proof exists in the literature that the Desarguesian plane is the only projective plane with 8 points per line (i.e., for the case $n=7$ ).

In the present paper, I apply the classical Kirkman Schoolgirl Problem to prove directly that the diagonals of a complete quadrilateral are never concurrent when $n=7 .^{2}$ In the paper that follows, Professor Hall uses my result to give a direct geometric proof of the theorem that any projective plane with 8 points per line is Desarguesian.

2. The impossibility of Fano's configuration. Suppose that Fano's configuration (i.e., a complete quadrilateral with concurrent diagonals) is contained in a projective plane with 8 points per line. The 7 points and 7 lines of this configuration will be called "the original points" and "the original lines." Besides the 7 original points, 35 new points lie on the 7 original lines. The whole plane has $1+7+7^{2}$ $=57$ points, so that exactly 15 points fail to lie on the original lines. I shall call these 15 points "the extra points." Through an original point $P$ there are 3 original lines and 5 new lines. Each new line through $P$ meets 3 original lines at $P$, meets the other 4 original lines at 4 distinct new points, and hence has exactly 3 extra points. The 15 extra points can be regarded as the "schoolgirls" of the Kirk-

Received by the editors February 17, 1953 and, in revised form, April 6, 1953.

${ }^{1}$ Sponsored by the Office of Ordnance Research, United States Army.

2 The Kirkman Problem: How can fifteen girls go walking every day for seven days, in five rows of three each, so that any two girls walk in the same row exactly once during the seven days? See [5]. 
man problem, the 5 new lines through $P$ inducing 5 rows of 3 "schoolgirls" each. The 7 original points induce 7 sets of 5 rows each, with every row having exactly 3 "schoolgirls." Regard each of these 7 sets as the Kirkman parade for one day. Such an interpretation will give a solution of the Kirkman problem if the unique line, $L$, through two distinct "extra points" is a new line through some original point. This is the case because $L$ meets the 7 original lines in at most 6 points.

Frank Nelson Cole has shown [6] that there are four types of Kirkman solutions (in Cole's terminology, I-II, III-IV, V-VI, VII). The first four "days" for each type are given by Columns $\alpha-\delta$ as follows:

$$
\text { Type (i) }
$$

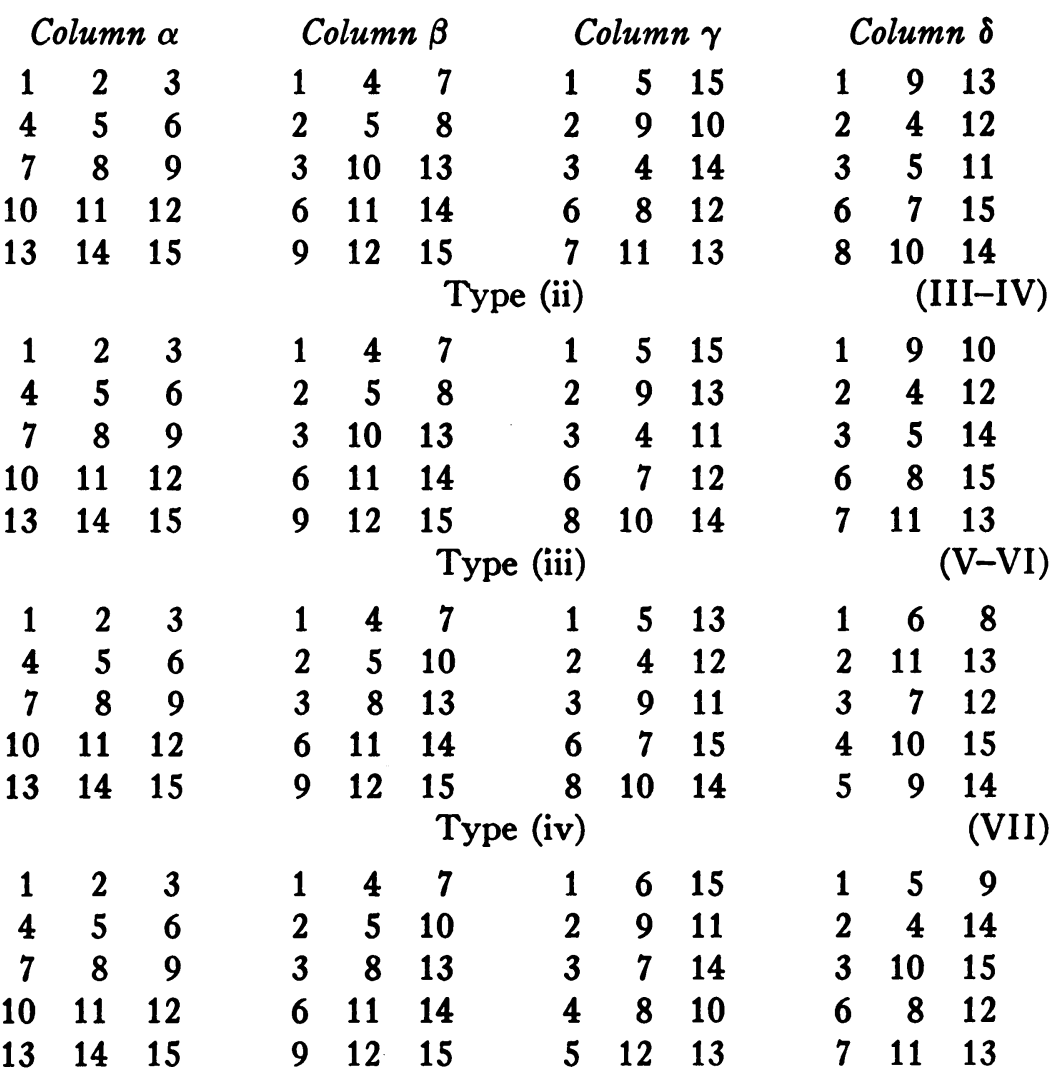

Denote by $A$ and $B$ those original points which determine the lacings of Columns $\alpha$ and $\beta$ respectively. (This means that the five new lines through $A$ have extra points $1,2,3 ; 4,5,6 ; 7,8,9 ; 10,11,12$; $13,14,15$; and the new lines through $B$ have the extra points 1, 4, 7; 
$6,11,14 ; 9,12,15 ; \cdots$ etc.) The Fano Configuration is, by itself, a Desarguesian plane and hence supports a collineation of itself which carries any noncollinear point-triple onto any noncollinear point-triple. Thus, if $C$ is an arbitrary original point not on line $A B$, it will be enough to check that $C$ determines the lacing of neither Column $\boldsymbol{\gamma}$ nor Column $\delta$.

LEMma. No generality is lost by assuming that Columns $\alpha, \beta, \gamma$ give the lacings of extra points from noncollinear original points $A, B, C$.

Proof. If Columns $\alpha, \beta, \gamma$ are the lacings from collinear original points, Columns $\alpha, \beta, \delta$ arise from noncollinear original points. In each of types (i), (ii), (iii), there is a permutation on the extra points which interchanges Columns $\alpha, \beta$, and which interchanges Columns $\boldsymbol{\gamma}, \boldsymbol{\delta}$. These permutations are:

$$
\begin{aligned}
& \text { Type (i) }(1,10)(2,13)(4,11)(5,14)(7,12)(8,15) . \\
& \text { Type (ii) }(1,13)(2,10)(4,14)(5,11)(7,15)(8,12) . \\
& \text { Type (iii) }(2,7)(3,4)(5,8)(6,13)(9,10)(11,15) .
\end{aligned}
$$

If Columns $\alpha, \beta, \gamma$ of type (iv) come from collinear original points, then Columns $\alpha, \beta, \delta$ arise from noncollinear points. However, Columns $\alpha, \beta, \delta$ of type (iv) differ in notation only, from Columns $\alpha, \beta, \gamma$ of type (iii) as can be seen by subjecting type (iv) to the permutation $(2,4)(3,7)(6,10)(9,13)(12,14)$.

Theorem. Fano's configuration is impossible in a plane with 8 points per projective line.

Proof. Assume the existence of Fano's configuration with original points $A-G$ and original lines $A B D, A C G, A E F, B E G, B C F, D F G$, $C D E$. The configuration determines a solution of the Kirkman problem, as explained above. By the lemma, we can assume that points $A, B, C$, determine the lacings of Columns $\alpha, \beta, \gamma$, respectively.

Each of the five new lines through $A$ has three extra points $\{1,2,3\},\{4,5,6\},\{7,8,9\},\{10,11,12\},\{13,14,15\}$. The new lines through $A$ meet $\{C, D\}$ in five distinct new points and meet $\{D, F\}$ in five distinct new points. The new lines through $B$ meet $\{C, D\}$ in five distinct new points and meet $\{D, F\}$ in five distinct new points. The new lines through $C$ also meet $\{D, F\}$ in five distinct points.

Assume, first, that the Kirkman solution is of Type (i). Then ${ }^{3}$ the point $\{D, F\} \cap\{4,5,6\}$ lies on line $\{9,12,15\}$ or on line $\{3,10,13\}$.

If $k$ and $m$ are lines, $k \bigcap m$ will denote the intersection of $k$ and $m$. 
Hence line $\{2,9,10\}$ cannot pass through $\{D, F\} \cap\{4,5,6\}$. Since, however, $\{2,9,10\}$ is a new line through $C,\{2,9,10\}$ must meet $\{D, F\}$ in a new point, and that new point is necessarily $\{D, F\}$ $\cap\{13,14,15\}$.

The point $\{D, F\} \cap\{1,2,3\}$ lies on one of the lines $\{6,11,14\}$, $\{9,12,15\}$, so that $\{6,8,12\}$ cannot pass through $\{D, F\} \cap$ $\{1,2,3\}$. Since $\{6,8,12\}$ intersects $\{D, F\}$ in some new point, $\{6,8,12\}$ passes through $\{D, F\} \cap\{13,14,15\}$. Thus points $C$ and $\{D, F\} \cap\{13,14,15\}$ are joined by the distinct lines $\{2,9,10\}$ and $\{6,8,12\}$. This contradicts the fact that two distinct points determine a unique line, and proves that the Kirkman solution is not of type (i).

Similarly, in type (ii), line $\{6,7,12\}$ cannot pass through $\{D, F\}$ $\cap\{1,2,3\}$ so must contain $\{D, F\} \cap\{13,14,15\}$; while $\{3,4,11$ cannot pass through $\{D, F\} \cap\{7,8,9\}$ so must contain $\{D, F\}$ $\cap\{13,14,15\}$. This is a contradiction, since $C$ is joined to $\{D, F\}$ $\cap\{13,14,15\}$ by both $\{6,7,12\}$ and $\{3,4,11\}$.

In type (iii), an identical argument shows that $\{1,5,13\}$ and $\{2,4,12\}$ would both join $C$ to $\{D, F\} \cap\{7,8,9\}$, proving (iii) impossible.

Assume, finally, that the Fano configuration leads to a Kirkman solution of type (iv). Then point $\{D, F\} \cap\{10,11,12\}$ lies on $\{3,8,13\}$ or $\{1,4,7\}$, whence line $\{3,7,14\}$ cannot contain point $\{D, F\} \cap\{10,11,12\}$, so must contain point $\{D, F\} \cap\{4,5,6\}$. This implies that point $\{D, F\} \cap\{4,5,6\}$ lies on $\{9,12,15\}$ rather than on $\{3,8,13\}$, so that $\{6,11,14\}$ passes through $\{C, D\}$ $\cap\{7,8,9\}$, forcing point $\{D, F\} \cap\{7,8,9\}$ to lie on $\{2,5,10\}$. It follows that $\{5,12,13\}$ cannot meet $\{D, F\}$ on $\{7,8,9\}$, and hence must be the line joining $C$ to point $\{D, F\} \cap\{1,2,3\}$. Since $\{4,8,10\}$ also passes through $C$, line $\{4,8,10\}$ must meet $\{D, F\}$ at $\{13,14,15\}$. This is impossible, because the point $\{D, F\} \cap$ $\{13,14,15\}$ lies on $\{1,4,7\}$ or $\{2,5,10\}$.

The proof of the theorem is now complete.

\section{Bibliography}

1. R. C. Bose, On the application of the properties of Galois fields to the problem of construction of hyper-Graeco-Latin squares, Sankhya, The Indian Journal of Mathematics vol. 3 (1938) pp. 328-338.

2. W. L. Stevens, The completely orthogonalised Latin squares, Annals of Eugenics vol. 9 (1939) pp. 82-93.

3. H. W. Norton, The $7 \times 7$ squares, Annals of Eugenics vol. 9 (1939) pp. 269-307.

4. R. C. Bose and K. R. Nair, On complete sets of Latin squares, Sankhya vol. 5, Part 4 (1941) pp. 361-382. 
5. T. P. Kirkman, Schoolgirl problem, The Lady's and Gentleman's Diary (1950).

6. F. N. Cole, Kirkman parades, Bull. Amer. Math. Soc. vol. 28 (1922) pp. 435437.

7. A. Sade, An omission in Norton's list of $7 \times 7$ squares, Ann. Math. Statist. vol. 22 (1951) pp. 306-307.

SYRACUSE UNIVERSITY

\section{UNIQUENESS OF THE PROJECTIVE PLANE WITH 57 POINTS}

MARSHALL HALL, JR.

1. Introduction. In the preceding paper, W. A. Pierce has given a new proof that Fano's configuration cannot exist in a projective plane with 8 points per line. In this paper, I shall use his result to give a proof of the theorem that any projective plane with 8 points per line is Desarguesian. (For historical notes, see the preceding paper.)

2. The proof. If $A_{1}, A_{2}, A_{3}, A_{4}$ form a quadrangle whose diagonal points are not collinear, then we have the following configuration:

$L_{1}: \quad A_{1} A_{2} B_{1} C_{5}$

$L_{2}: A_{1} A_{3} B_{2} C_{3}$

$L_{3}: A_{1} A_{4} B_{3} C_{1}$

$L_{4}: \quad A_{2} A_{3} B_{3} C_{2}$

$L_{5}: A_{2} A_{4} B_{2} C_{4}$

$L_{6}: A_{3} A_{4} B_{1} C_{6}$

$M_{1}: \quad B_{1} B_{2} C_{1} C_{2}$

$M_{2}: \quad B_{1} B_{8} C_{8} C_{4}$

$M_{8}: \quad B_{2} B_{8} C_{6} C_{6}$

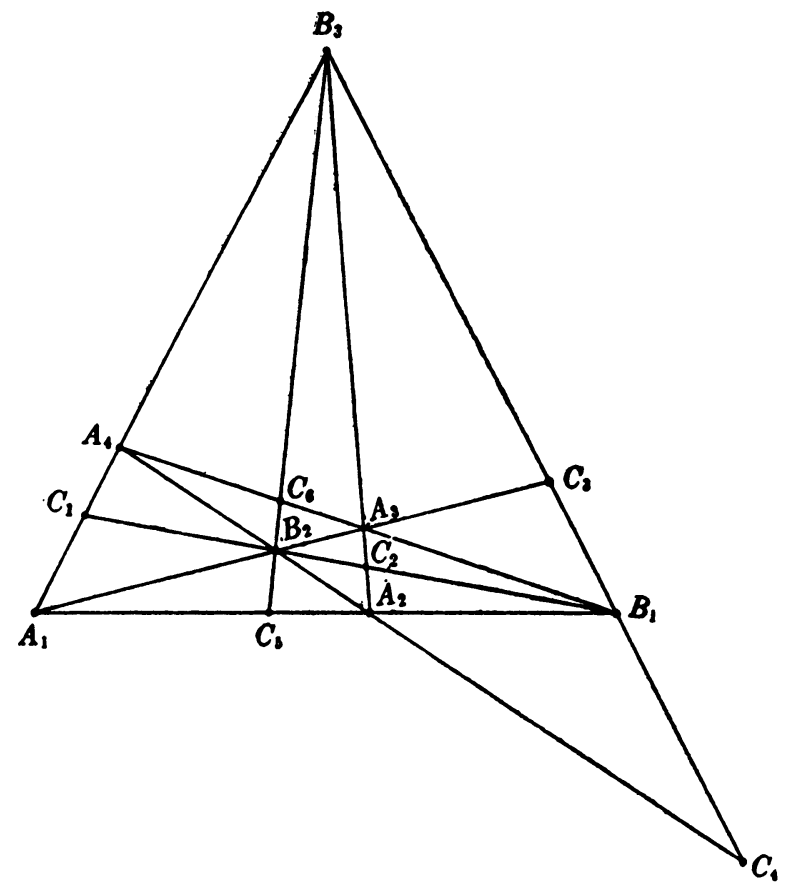

Presented to the Society, April 24, 1953; received by the editors February 17, 1953. 